\title{
Use of Traditional Chinese Medicine and Its Impact on Medical Cost among Urban Ischemic Stroke Inpatients in China: A National Cross-Sectional Study
}

\author{
Zhengwei Huang $\mathbb{D}^{\mathbb{D}},{ }^{1}$ Xuefeng Shi $\mathbb{D}^{1,},{ }^{1,2}$ Stephen Nicholas $\mathbb{D}^{3},{ }^{3,4,5,6}$ Elizabeth Maitland ${ }^{\mathbb{D}}{ }^{7}$ \\ Yong Yang $\mathbb{\oplus},^{8}$ Weihan Zhao $\mathbb{D}^{1},{ }^{1}$ Yong $M a,{ }^{9}$ and Yan Jiang $\mathbb{1}^{1}$ \\ ${ }^{1}$ School of Management, Beijing University of Chinese Medicine, Beijing, China \\ ${ }^{2}$ National Institute of Traditional Chinese Medicine Strategy and Development, Beijing University of Chinese Medicine, \\ Beijing, China \\ ${ }^{3}$ Australian National Institute of Management and Commerce, Eveleigh Sydney, NSW 2015, Australia \\ ${ }^{4}$ School of Economics and School of Management, Tianjin Normal University, Tianjin, China \\ ${ }^{5}$ Guangdong Institute for International Strategies, Guangdong University of Foreign Studies, Guangzhou, China \\ ${ }^{6}$ Newcastle Business School, University of Newcastle, Newcastle, Callaghan, Australia \\ ${ }^{7}$ School of Management, University of Liverpool, Chatham Building, Chatham Street, Liverpool L697ZH, UK \\ ${ }^{8}$ Medical Device Regulatory Research and Evaluation Center, West China Hospital, Sichuan University, Chengdu, China \\ ${ }^{9}$ China Health Insurance Research Association, Beijing, China
}

Correspondence should be addressed to Yan Jiang; jy_ftn@163.com

Received 13 August 2021; Accepted 12 October 2021; Published 29 October 2021

Academic Editor: Harry Lee

Copyright (c) 2021 Zhengwei Huang et al. This is an open access article distributed under the Creative Commons Attribution License, which permits unrestricted use, distribution, and reproduction in any medium, provided the original work is properly cited.

Background. Traditional Chinese medicine (TCM) has long been widely adopted by the Chinese people and has been covered by China's basic medical insurance schemes to treat ischemic stroke. Previous research has mainly highlighted the therapy effect of TCM on ischemic stroke patients. Some studies have demonstrated that employing TCM can reduce the medical burden on other diseases. But no research has explored whether using TCM could reduce inpatient medical cost for ischemic stroke in mainland China. The purpose of this study is to investigate the impact of the use of TCM on the total inpatient cost of ischemic stroke and to explore whether TCM has played the role of being complementary to, or an alternative for, conventional medicine to treat ischemic stroke. Methods. We conducted a national cross-sectional analysis based on a 5\% random sample from claims data of China Urban Employee Basic Medical Insurance (UEBMI) and Urban Resident Basic Medical Insurance (URBMI) schemes in 2015. Mann-Whitney test was used to compare unadjusted total inpatient cost, conventional medication cost, and nonpharmacy cost estimates. Ordinary least square regression analysis was performed to compare demographics-adjusted total inpatient cost and to examine the association between TCM cost and conventional medication cost. Results. A total of 47321 urban inpatients diagnosed with ischemic stroke were identified in our study, with 92.6\% (43843) of the patients using TCM in their inpatient treatment. Total inpatient cost for TCM users was significantly higher than TCM nonusers (USD 1217 versus USD 1036, $P<0.001$ ). Conventional medication cost was significantly lower for TCM users (USD 335 versus USD 436, $P<0.001$ ). The average cost of TCM per patient among TCM users was USD 289. Among TCM users, conventional medication costs were found to be positively associated with TCM cost after adjusting for confounding factors (Coef. $=0.144, P<0.001$ ). Conclusion. Although the use of TCM reduced the cost of conventional medicine compared with TCM nonusers, TCM imposed an extra financial component on the total inpatient cost on TCM users. Our study suggests that TCM mainly played a complementary role to conventional medicine in ischemic stroke treatment in mainland China. 


\section{Background}

Stroke is one of the world's most serious chronic diseases due to the severity of its health damage and to its catastrophic health costs. In 2017, there were approximately 104 million stroke patients, with an estimated 13 million new global cases in 2020. Stroke claimed over 6 million lives in 2017, becoming the third leading cause of both death and disability-adjusted life-years (DALYs) $[1,2]$. The health care expenditure spent on stroke imposed a significant economic burden on health systems, estimated at over $€ 27$ billion in Europe alone in 2020 [3], and imposed a heavy financial burden on families with stroke victims, especially in lowand middle-income countries [1].

Stroke has been the leading cause of both death and DALYs in China, with an estimated 1.1 million stroke-related deaths in 2013 or about 15\% of the global total of 6 million stroke deaths $[4,5]$. Among various types of stroke, ischemic was the main subtype in China [6]. A recent study revealed that the weighted average 2013-2016 annual total medical cost of stroke was RMB10637 (USD1707.82), with inpatient costs accounting for 94\% (RMB10034 or USD1586) of total stoke medical costs, with medication costs accounting for $50.6 \%$ of average stroke-related medical costs [6]. While Chinese stroke patients mainly receive conventional modern medicine in their treatment, it has been claimed that conventional medicine fails to some degree to optimally treat or reduce costs of stroke [7-9].

Traditional, complementary, and alternative medicine (TCAM) is regarded as both a complement and an alternative to conventional stroke treatment for its equitability, accessibility, and low cost. Previous studies show that $46 \%$ of stroke patients in the United States [10], 66\% in Malaysia [11], 36\% in Indian [12], and 54\% in Korea [13] have used TCAM stroke treatments. Evidence suggests that more than half of stroke patients who received TCAM treatment benefited, including improved stroke symptoms, walking patterns, psychological distress, limb strength, and standing balance $[12,14]$. Traditional Chinese medicine (TCM), one specific type of TCAM, has been used for the treatment of stroke in China for thousands of years, due to its safety, lower cost, effectiveness, and convenience [15]. The Healthy China 2030 Program has stressed the uniqueness and importance of TCM in prevention, rehabilitation, and treatment for severe diseases, including stroke. Studies have shown that TCM stroke treatment can promote angiogenesis, strengthen neurological function, improve stroke recovery, advance life quality, and lower recurrence risk through its nonmedication therapies, herbal medicines, and pharmaceutical preparations [5, 16-25]. Using a Taiwanese National Health Insurance (NHI) database, Su et al. [26] found that, among patients with uterine fibroid, TCM use correlated negatively with consumption of conventional medicine and decreased total medical costs. Tsai et al. [27] also demonstrated that, among patients with heart failure, the hospital cost was lower for TCM users than for TCM nonusers. Both studies suggested that TCM could be a substitute for conventional medicine to treat diseases and with lower healthcare costs.
Given the partial evidence of lower cost and effectiveness of TCM ischemic stroke treatment, do TCM stroke treatment users consume fewer conventional medications and incur lower medical cost than conventional-only stroke treatment users? Unfortunately, few studies have investigated the impact of the use of TCM on medical cost in mainland China. This study investigates the impact of the use of TCM on total inpatient medical costs of ischemic stroke sufferers and explores whether TCM was a complement or an alternative for conventional ischemic stroke treatments from a cost perspective.

\section{Materials and Methods}

2.1. Data Source. Our data come from a 5\% random sample of the Urban Employee Basic Medical Insurance (UEBMI) and the Urban Resident Basic Medical Insurance (URBMI) beneficiaries' insurance claims, which used a stratified random sample design and were conducted in 2015 by China Health Insurance Research Association (CHIRA) [28]. UEBMI and URBMI were the two main social health insurance schemes administered by China government, covering more than $95 \%$ of the urban residents, for roughly 750 million, or 53\%, of the total Chinese population in 2015 $[29,30]$. Based on contributions from employers and employees, UEBMI, launched in 1998, offers compulsory and comprehensive insurance for employed people with a per capita fund of RMB3144 (USD505) in 2015, with an $80 \%$ reimbursement rate and basic urban wage reimbursement ceiling. Based on a government subsidy and individual premiums, URBMI, started in 2007, is voluntary, offering limited insurance to the unemployed, retired, students, and children among urban residents, with a per capita fund of RMB560 (USD 90) in 2015, with a 70\% reimbursement rate and farmer income-based reimbursement ceiling [31]. These cross-sectional data consist of ischemic stroke patients' demographic information (sex and age), diagnosis (classified according to International Classification of Diseases, 10th edition (ICD-10)), length of hospital stay (LOS), hospital level (primary, secondary, and tertiary), hospital type (TCM and non-TCM), economic region (east, west, and central), type of medical insurance expenditure (URBMI and UEBMI), and inpatient cost of hospital treatment.

2.2. Samples. According to the International Classification of Diseases, 10th version (ICD-10) codes, all patients whose primary diagnosis was ischemic stroke (ICD-10: I63.X) between January 2015 and December 2015 were included. Inclusion criteria for the inpatients with ischemic stroke were at least one inpatient claim with a primary diagnosis of ICD-10: I63.X code (defined as the "ischemic condition of the brain, producing a persistent focal neurological deficit in the area of distribution of the cerebral arteries" [32]) in 2015. Patients were excluded when their data were incomplete or logically erroneous when calculating medical costs, such as when individual total medical costs were not equal to the sum of medication cost and nonpharmacy cost. We 
identified 47321 ischemic stroke patients in the 2015 CHIRA claims database.

2.3. Measures. According to the CHIRA claims data, TCM treatment included three subtypes, Chinese herbal medicine $(\mathrm{H})$, Chinese patent medicine $(\mathrm{P})$, and Chinese medicine injection $(\mathrm{J})$. TCM users were defined by inpatients who utilized any kind of the three TCM treatments. TCM users frequently received conventional medicines as well as TCM treatments. TCM nonusers were defined as patients who did not receive any type of TCM treatment. We also extracted information on 8 prevalent comorbidities among ischemic stroke patients [33], comprising hypertension, coronary heart disease, diabetes, chronic pulmonary disease, peripheral vascular disease, other neurological diseases, rheumatoid arthritis, and peptic ulcer disease.

Medical inpatient costs comprised conventional medication pharmacy costs, TCM medication pharmacy costs, and nonpharmacy costs. Except for the costs of TCM and conventional prescription drugs, the nonpharmacy cost referred to all other inpatient costs, including diagnostic tests and nonmedication therapy. The inpatient cost was calculated for each patient for the whole year of 2015. When a patient was admitted more than once during 2015, costs for each admission were summed to obtain the inpatient cost. All patients leaving hospital from January 1, 2015, to December 31, 2015, were considered as the index event. Given the data source, an individual's 2014 hospital costs before the index event were included in an individual's 2015 hospital costs. All costs were based on a constant 2015 US $\$ 1.0=$ RMB 6.2284 annual average exchange rate.

2.4. Statistical Analysis. Descriptive statistics (percentage and median with interquartile range (IQR)) were used to describe the demographic information and hospital costs. Demographic variables and comorbidities between TCM users and TCM nonusers were examined using chi-square tests. Since the value of medical expenditures usually has a skewed distribution, the Mann-Whitney test was used to identify the differences in inpatient costs between TCM users and TCM nonusers. To quantify the difference in total inpatient cost between TCM users and TCM nonusers, an ordinary least squares regression analysis was conducted, in which the dependent variable was the logarithm of total inpatient cost and the independent variable was the use/ nonuse of TCM for each patient, adjusted for the confounding variables, including length of hospital stay (LOS), sex, age, type of medical insurance, hospital level, type of hospital, region, and comorbidities, which can influence both the dependent and independent variable. A further ordinary least squares regression analysis was performed to investigate the association between TCM cost and conventional medication cost, with the logarithm of TCM cost as the dependent variable and logarithm of conventional medication cost as the independent variable of interest, adjusted for the confounding factors described above. A two-sided $P$ value less than 0.05 was considered statistically significant. All analyses were performed using STATA/SE 15.

\section{Results}

3.1. Basic Characteristics. As shown in Table 1, 47321 patients suffered ischemic stroke, where 7.4\% (3478) did not use any TCM treatment (TCM nonusers) and 92.6\% (43843) used at least one type of TCM treatment (TCM users), comprising Chinese herbal medicine, Chinese patent medicine, and Chinese medicine injection. Table 1 shows that $56.6 \%$ (26795) of ischemic stroke inpatients were male, but the proportion of female TCM users (43.7\%) was significantly higher than TCM nonusers $(39.7 \%)(P<0.001)$. The median age of our sample was 68 years; $63.3 \%$ (29950) of patients participated in the UEBMI scheme; $14.8 \%$ of patients received medical treatment in TCM hospitals, but significantly more TCM users were treated in TCM hospitals $(15.1 \%)$ than TCM nonusers $(11.2 \%) \quad(P<0.001)$. From Table 1, 31.7\% of TCM users were treated in tertiary hospitals and $19.6 \%$ in primary hospitals, while $39.7 \%$ of TCM nonusers were treated in tertiary and only $14.4 \%$ in primary hospitals $(P<0.001)$. TCM users were mainly from the central region $(46.7 \%)$, followed by the eastern region (34.7\%), but TCM nonusers were roughly equally treated in the central $(42.8 \%)$ and eastern $(40.5 \%)$ regions $(P<0.001)$. The proportions of patients who had comorbidities, such as hypertension, coronary heart disease, diabetes, chronic pulmonary disease, peripheral vascular disease, other neurological diseases, rheumatoid arthritis, and peptic ulcer disease, were all significantly higher among TCM users than TCM nonusers $(P<0.001)$. Finally, the average length of stay (ALOS) for TCM users (15.1 days) was significantly lower than TCM nonusers $(17.7$ days $)(P<0.001)$.

\subsection{Inpatient Cost between TCM Users and TCM Nonusers} Associated with Demographic Characteristics. Table 2 reveals the differences in TCM user and TCM nonuser ischemic stroke inpatient medical costs. Overall, the median total inpatient cost for TCM users was RMB7579 (USD1217), significantly higher than TCM nonusers (RMB6455 (USD1036) $(P<0.001)$. TCM users' significantly higher inpatient cost than TCM nonusers differed according to sex, insurance type, TCM hospital, and region (all $P<0.001$ ). Inpatient cost of TCM users over 30 years old and TCM users admitted to secondary hospitals and tertiary hospitals had significantly higher medical costs than TCM nonusers. In addition, TCM users with comorbidities of hypertension, coronary heart disease, diabetes, and other neurological diseases had significantly higher inpatient cost than TCM nonusers.

\subsection{Analysis of Inpatient TCM Users and TCM Nonusers} Medical Costs. To further examine the difference in inpatient medical cost between TCM users and TCM nonusers, we conducted a multivariate regression analysis after the confounding variables were fixed. Table 3 reveals that TCM users incurred a 32.5\% (Coef. $=0.325, P<0.001$ ) higher 
TABLE 1: Sample characteristics of TCM users and TCM nonusers among stroke patients.

\begin{tabular}{|c|c|c|c|c|c|}
\hline \multicolumn{2}{|c|}{ Characteristics } & \multirow{2}{*}{$\begin{array}{c}\text { Overall } \\
47321(100.0)\end{array}$} & \multirow{2}{*}{$\begin{array}{c}\text { TCM nonusers } \\
3478(7.4)\end{array}$} & \multirow{2}{*}{$\begin{array}{c}\text { TCM users } \\
43843(92.6)\end{array}$} & \multirow{2}{*}{$P$ value } \\
\hline Number of patients, $n(\%)$ & & & & & \\
\hline \multirow{2}{*}{ Sex, $n(\%)$} & Male & $26795(56.6)$ & $2098(60.3)$ & $24697(56.3)$ & $<0.001$ \\
\hline & Female & $20526(43.4)$ & $1380(39.7)$ & $19146(43.7)$ & \\
\hline \multirow[t]{3}{*}{ Age (years), median (IQR) } & & $68(61-77)$ & $68(60-77)$ & $68(61-76)$ & 0.565 \\
\hline & $0-29$ & $55(0.1)$ & $12(0.3)$ & $43(0.1)$ & $<0.001$ \\
\hline & $30-44$ & $921(1.9)$ & $85(2.4)$ & $836(1.9)$ & \\
\hline \multirow{4}{*}{ Age group, $n(\%)$} & $45-59$ & $9245(19.5)$ & $697(20.0)$ & $8548(19.5)$ & \\
\hline & $60-69$ & $14974(31.6)$ & $1061(30.5)$ & $13913(31.7)$ & \\
\hline & $70-79$ & $14528(30.7)$ & $962(27.7)$ & $13566(30.9)$ & \\
\hline & $\geqq 80$ & $7598(16.1)$ & $661(19.0)$ & $6937(15.8)$ & \\
\hline \multirow{3}{*}{ Insurance type, $n(\%)$} & UEBMI & $29950(63.3)$ & $2456(70.6)$ & $27494(62.7)$ & $<0.001$ \\
\hline & URBMI & $17371(36.7)$ & $1022(29.4)$ & $16349(37.3)$ & \\
\hline & Primary & $9078(19.2)$ & $500(14.4)$ & $8578(19.6)$ & $<0.001$ \\
\hline \multirow[t]{2}{*}{ Hospital level, $n(\%)$} & Secondary & $22957(48.5)$ & $1596(45.9)$ & $21361(48.7)$ & \\
\hline & Tertiary & $15286(32.3)$ & $1382(39.7)$ & $13904(31.7)$ & \\
\hline \multirow{3}{*}{ TCM hospital, $n(\%)$} & Yes & $7000(14.8)$ & $388(11.2)$ & $6612(15.1)$ & $<0.001$ \\
\hline & No & $40321(85.2)$ & $3090(88.8)$ & $37231(84.9)$ & \\
\hline & East & $16637(35.2)$ & $1409(40.5)$ & $15228(34.7)$ & $<0.001$ \\
\hline \multirow[t]{5}{*}{ Region, $n(\%)$} & Central & $21970(46.4)$ & $1488(42.8)$ & $20482(46.7)$ & \\
\hline & West & $8714(18.4)$ & $581(16.7)$ & $8133(18.6)$ & \\
\hline & Hypertension & $2458(5.2)$ & $92(2.6)$ & $2366(5.4)$ & $<0.001$ \\
\hline & Coronary heart disease & $1558(3.3)$ & $44(1.3)$ & $1514(3.5)$ & $<0.001$ \\
\hline & Diabetes & $868(1.8)$ & $44(1.3)$ & $824(1.9)$ & 0.009 \\
\hline \multirow{5}{*}{ Comorbidity, $n$ (\%) } & Chronic pulmonary disease & $394(0.8)$ & $13(0.4)$ & $381(0.9)$ & 0.002 \\
\hline & Peripheral vascular disease & $177(0.4)$ & $7(0.2)$ & $170(0.4)$ & 0.083 \\
\hline & Other neurological diseases & $168(0.4)$ & $5(0.1)$ & $163(0.4)$ & 0.03 \\
\hline & Rheumatoid arthritis & $91(0.2)$ & $7(0.2)$ & $84(0.2)$ & 0.9 \\
\hline & Peptic ulcer disease & $87(0.2)$ & $0(0.0)$ & $87(0.2)$ & 0.009 \\
\hline Average length of stay (ALOS) & & 15.3 & 17.7 & 15.1 & $<0.001$ \\
\hline
\end{tabular}

$P$ values are based on the chi-square test and Mann-Whitney test; TCM: traditional Chinese medicine, UEBMI: Urban Employee Basic Medical Insurance scheme, URBMI: Urban Resident Basic Medical Insurance scheme, and IQR: interquartile range.

medical cost compared to TCM nonusers after adjusting for length of stay (LOS), age, sex, insurance type, hospital type, region, and comorbidities.

3.4. Composition of TCM Users and TCM Nonusers Inpatient Medical Costs. Table 4 compares the composition of inpatient cost of TCM nonusers and TCM users, which helps explain the reason why TCM users faced a higher inpatient medical cost component. As categorized in the insurance payment system, inpatient medical cost for ischemic stroke inpatients were divided into conventional medication costs, TCM medication costs, and nonpharmacy medical costs. From the payment data in Table 4, there was no significant difference in nonpharmacy medical cost between TCM users and TCM nonusers $(P>0.290)$. While ischemic stroke TCM users incurred RMB629/USD101 lower conventional medication cost (RMB2087/USD335) compared to TCM nonusers (RMB2716/USD436) $(P<0.001)$, TCM medication costs (RMB1803/USD289) meant that total medication costs of TCM users (RMB4175/USD670) were higher than TCM nonusers medication costs (RMB2716/USD436) $(P<0.001)$.

3.5. Multivariate Regression Analysis to Examine the Association of TCM Cost. Table 4 shows that the use of TCM imposed a substantial medical cost component on TCM users. A multiple regression analysis in Table 5 modeled the association between TCM costs and conventional medication costs for TCM users, which showed a positive association of TCM costs with conventional medication costs (Coef. $=0.144, P<0.001$ ). Among TCM users, TCM medication cost significantly increased with the prescription of conventional medicine, demonstrating that TCM treatments were complements, rather than substitutes, to conventional treatment.

\section{Discussion}

Traditional Chinese medicine (TCM) was a treatment sought out by ischemic stroke patients and made available by healthcare providers. To our knowledge, this is the first study using a large nationwide Chinese health insurance claims database to explore whether using TCM could reduce medical cost for ischemic stroke inpatients. Compared with patients who did not use any kind of TCM (7.4\%), the majority of ischemic stroke patients utilized some TCM treatments $(92.6 \%)$, which was much higher than TCM use in other countries that used TCM in the stroke treatment [10-13]. Also, complementary and alternative medications were less expensive than conventional medications $[26,34,35]$. While TCM users incurred lower conventional medication costs than TCM nonusers, we found that 
TABLE 2: Inpatient medical cost TCM users and TCM nonusers.

\begin{tabular}{|c|c|c|c|c|c|c|}
\hline \multirow{2}{*}{\multicolumn{2}{|c|}{ Characteristics }} & \multicolumn{2}{|c|}{ TCM nonusers } & \multicolumn{2}{|c|}{ TCM users } & \multirow{2}{*}{$P$ value } \\
\hline & & Median & IQR & Median & IQR & \\
\hline \multirow{2}{*}{ Sex } & Male & 6766 & $(3599-12565)$ & 8032 & $(4602-14418)$ & $<0.001$ \\
\hline & Female & 6046 & $(3371-11126)$ & 7090 & $(4117-12300)$ & $<0.001$ \\
\hline \multirow{6}{*}{ Age group } & $0-29$ & 7968 & $(6630-12072)$ & 10348 & $(4775-19859)$ & 0.299 \\
\hline & $30-44$ & 5746 & $(3257-11238)$ & 7988 & $(4195-14657)$ & 0.013 \\
\hline & $45-59$ & 6468 & $(3571-12120)$ & 7395 & $(4210-12867)$ & $<0.001$ \\
\hline & $60-69$ & 6061 & $(3336-10996)$ & 7092 & $(4134-12338)$ & $<0.001$ \\
\hline & $70-79$ & 6207 & (3323-11153) & 7751 & $(4522-13547)$ & $<0.001$ \\
\hline & $\geqq 80$ & 7583 & $(4031-15621)$ & 8667 & $(4813-16331)$ & 0.001 \\
\hline \multirow{3}{*}{ Insurance type } & UEBMI & 7361 & $(4039-13210)$ & 9091 & $(5432-15839)$ & $<0.001$ \\
\hline & URBMI & 4538 & $(2541-8500)$ & 5524 & $(3213-9582)$ & $<0.001$ \\
\hline & Primary & 3570 & $(1776-9283)$ & 3516 & $(2115-6070)$ & 0.588 \\
\hline \multirow[t]{2}{*}{ Hospital level } & Secondary & 4883 & $(3040-8539)$ & 6982 & $(4488-11116)$ & $<0.001$ \\
\hline & Tertiary & 9595 & $(5971-15471)$ & 12685 & $(7977-20786)$ & $<0.001$ \\
\hline \multirow{3}{*}{ Admission to TCM hospital } & Yes & 4663 & $(3117-8237)$ & 9424 & $(5909-16751)$ & $<0.001$ \\
\hline & No & 6786 & $(3574-12376)$ & 7275 & $(4157-12840)$ & $<0.001$ \\
\hline & East & 5086 & $(2050-10609)$ & 6948 & $(4025-12386)$ & $<0.001$ \\
\hline \multirow[t]{5}{*}{ Region } & Central & 4928 & $(3150-8784)$ & 6386 & $(3870-10606)$ & $<0.001$ \\
\hline & West & 9092 & $(5143-15545)$ & 10351 & $(5882-17599)$ & $<0.001$ \\
\hline & Hypertension & 7619 & $(4982-18733)$ & 12178 & $(7197-21065)$ & 0.001 \\
\hline & Coronary heart disease & 7286 & $(3791-13955)$ & 12497 & $(7199-22242)$ & $<0.001$ \\
\hline & Diabetes & 7696 & $(4494-22358)$ & 14412 & $(8725-25169)$ & 0.022 \\
\hline \multirow{5}{*}{ Comorbidity } & Chronic pulmonary disease & 12431 & $(6664-23773)$ & 17396 & $(9646-35066)$ & 0.279 \\
\hline & Peripheral vascular disease & 12708 & $(4163-35509)$ & 14008 & $(9152-25808)$ & 0.572 \\
\hline & Other neurological diseases & 4163 & $(2627-6994)$ & 12976 & $(8433-19486)$ & 0.002 \\
\hline & Rheumatoid arthritis & 6256 & $(2605-7141)$ & 7388 & $(4004-13998)$ & 0.14 \\
\hline & Peptic ulcer disease & - & - & 16816 & $(10206-25507)$ & - \\
\hline
\end{tabular}

$P$ values are based on the Mann-Whitney test; TCM: traditional Chinese medicine; UEBMI: Urban Employee Basic Medical Insurance scheme; URBMI: Urban Resident Basic Medical Insurance scheme; IQR: Interquartile range.

TABLE 3: Multiple regression analysis for total inpatient costs.

\begin{tabular}{|c|c|c|c|c|c|}
\hline \multirow{2}{*}{\multicolumn{2}{|c|}{ Parameters }} & \multirow[t]{2}{*}{ Coef. } & \multirow[t]{2}{*}{$P>z$} & \multicolumn{2}{|c|}{$\begin{array}{l}\text { 95\% Wald confidence } \\
\text { interval }\end{array}$} \\
\hline & & & & Lower & Upper \\
\hline Use of TCM & TCM users & 0.325 & $<0.001$ & 0.304 & 0.347 \\
\hline Length of stay & & 0.756 & $<0.001$ & 0.748 & 0.764 \\
\hline Age & & 0.002 & $<0.001$ & 0.002 & 0.003 \\
\hline Sex & Male & 0.026 & $<0.001$ & 0.015 & 0.037 \\
\hline Insurance type & UEBMI & 0.109 & $<0.001$ & 0.097 & 0.121 \\
\hline \multirow{2}{*}{ Hospital type } & Tertiary hospital & 1.001 & $<0.001$ & 0.984 & 1.018 \\
\hline & Secondary hospital & 0.535 & $<0.001$ & 0.520 & 0.550 \\
\hline Admission to TCM hospitals & & -0.006 & 0.458 & -0.022 & 0.010 \\
\hline \multirow{2}{*}{ Region } & East & 0.217 & $<0.001$ & 0.201 & 0.234 \\
\hline & Central & -0.108 & $<0.001$ & -0.124 & -0.093 \\
\hline Hypertension & & 0.054 & 0.001 & 0.023 & 0.085 \\
\hline Coronary heart disease & & 0.054 & 0.005 & 0.016 & 0.092 \\
\hline Diabetes & & 0.114 & $<0.001$ & 0.068 & 0.159 \\
\hline Chronic pulmonary disease & & 0.364 & $<0.001$ & 0.301 & 0.427 \\
\hline Peripheral vascular disease & & 0.080 & 0.091 & -0.013 & 0.172 \\
\hline Other neurological diseases & & -0.014 & 0.781 & -0.110 & 0.083 \\
\hline Rheumatoid arthritis & & 0.034 & 0.600 & -0.092 & 0.160 \\
\hline Peptic ulcer disease & & 0.112 & 0.096 & -0.020 & 0.244 \\
\hline _Cons & & 5.959 & $<0.001$ & 5.912 & 6.006 \\
\hline
\end{tabular}

$R$-square $=0.5780$ and adjusted R-square $=0.5779$ in a multiple linear regression model that was adjusted for length of stay, age, sex, insurance type, hospital type, region, hypertension, coronary heart disease, diabetes, chronic pulmonary disease, peripheral vascular disease, other neurological diseases, rheumatoid arthritis, and peptic ulcer disease. The baseline represents the inpatient cost for a female who did not use any TCM with Urban Resident Basic Medical Insurance admitted to a primary hospital and non-TCM hospital in the western region without the above-observed comorbidities. TCM: traditional Chinese medicine; UEBMI: Urban Employee Basic Medical Insurance scheme; URBMI: Urban Resident Basic Medical Insurance scheme. 
TABLE 4: Composition of medication and medical cost TCM users and TCM nonusers.

\begin{tabular}{|c|c|c|c|c|}
\hline \multicolumn{2}{|l|}{ Variables } & TCM nonusers & TCM users & $P$ value \\
\hline Total medical cost & $\begin{array}{l}\text { Median } \\
\text { IQR }\end{array}$ & $\begin{array}{c}6455 \\
(3494-11932)\end{array}$ & $\begin{array}{c}7579 \\
(4364-13404)\end{array}$ & $<0.001$ \\
\hline Total medication cost & $\begin{array}{c}\text { Median } \\
\text { IQR } \\
\text { Percentage }\end{array}$ & $\begin{array}{c}2716 \\
(1155-5463) \\
45.1\end{array}$ & $\begin{array}{c}4175 \\
(2261-7783) \\
54.6\end{array}$ & $<0.001$ \\
\hline Conventional medication cost & $\begin{array}{c}\text { Median } \\
\text { IQR } \\
\text { Percentage }\end{array}$ & $\begin{array}{c}2716 \\
(1155-5463) \\
45.1\end{array}$ & $\begin{array}{c}2087 \\
(865-4602) \\
32.7\end{array}$ & $<0.001$ \\
\hline TCM cost & $\begin{array}{c}\text { Median } \\
\text { IQR } \\
\text { Percentage }\end{array}$ & $\begin{array}{l}- \\
-\end{array}$ & $\begin{array}{c}1803 \\
(810-3316) \\
21.9\end{array}$ & $<0.001$ \\
\hline Nonpharmacy cost & $\begin{array}{c}\text { Median } \\
\text { IQR } \\
\text { Percentage }\end{array}$ & $\begin{array}{c}3347 \\
(1653-6235) \\
54.9\end{array}$ & $\begin{array}{c}3173 \\
(1720-5703) \\
45.4\end{array}$ & 0.290 \\
\hline
\end{tabular}

$P$ values are based on the Mann-Whitney test; TCM: traditional Chinese medicine; IQR: interquartile range.

TABLE 5: Multiple regression analysis for TCM cost.

\begin{tabular}{|c|c|c|c|c|c|}
\hline \multirow{2}{*}{\multicolumn{2}{|c|}{ Parameters }} & \multirow[t]{2}{*}{ Coef. $^{a}$} & \multirow[t]{2}{*}{$P>z$} & \multicolumn{2}{|c|}{$\begin{array}{l}\text { 95\% Wald confidence } \\
\text { interval }\end{array}$} \\
\hline & & & & Lower & Upper \\
\hline Conventional medication cost & & 0.144 & $<0.001$ & 0.132 & 0.156 \\
\hline Length of stay & & 0.636 & $<0.001$ & 0.616 & 0.657 \\
\hline Age & & -0.002 & $<0.001$ & -0.003 & -0.001 \\
\hline Sex & Male & -0.004 & 0.769 & -0.027 & 0.020 \\
\hline Insurance type & UEBMI & 0.109 & $<0.001$ & 0.083 & 0.134 \\
\hline \multirow{2}{*}{ Hospital type } & Tertiary hospital & -0.012 & 0.542 & -0.052 & 0.027 \\
\hline & Secondary hospital & -0.113 & $<0.001$ & -0.147 & -0.080 \\
\hline Admission to TCM hospitals & & 0.225 & $<0.001$ & 0.192 & 0.258 \\
\hline \multirow{2}{*}{ Region } & East & -0.006 & 0.712 & -0.040 & 0.028 \\
\hline & Central & -0.129 & $<0.001$ & -0.161 & -0.097 \\
\hline Hypertension & & -0.045 & 0.162 & -0.109 & 0.018 \\
\hline Coronary heart disease & & 0.261 & $<0.001$ & 0.183 & 0.338 \\
\hline Diabetes & & 0.068 & 0.159 & -0.027 & 0.162 \\
\hline Chronic pulmonary disease & & -0.120 & 0.068 & -0.249 & 0.009 \\
\hline Peripheral vascular disease & & 0.111 & 0.251 & -0.078 & 0.299 \\
\hline Other neurological diseases & & -0.189 & 0.059 & -0.385 & 0.007 \\
\hline Rheumatoid arthritis & & -0.134 & 0.319 & -0.397 & 0.129 \\
\hline Peptic ulcer disease & & -0.474 & $<0.001$ & -0.738 & -0.209 \\
\hline _Cons & & 4.771 & $<0.001$ & 4.670 & 4.872 \\
\hline
\end{tabular}

${ }^{a} R$-square $=0.1893$ and adjusted $R$-square $=0.1889$ in a multiple linear regression model that was adjusted for length of stay, age, sex, insurance type, hospital type, region, hypertension, coronary heart disease, diabetes, chronic pulmonary disease, peripheral vascular disease, other neurological diseases, rheumatoid arthritis, and peptic ulcer disease. The baseline represents the TCM cost for a female with Urban Resident Basic Medical Insurance admitted to a primary hospital and non-TCM hospital in the western region without the above-observed comorbidities. TCM: traditional Chinese medicine; UEBMI: Urban Employee Basic Medical Insurance scheme; URBMI: Urban Resident Basic Medical Insurance scheme.

inpatient TCM users' medical costs were $32.5 \%$ higher than those of TCM nonusers after adjusting for LOS, age, sex, insurance type, hospital type, region, and comorbidities.

While total inpatient medical cost was significantly higher for TCM users, we found that the use of TCM reduced on average the cost of conventional medication use by RMB629/USD101 compared with TCM nonusers. This small TCM impact on conventional medication costs suggests that TCM attenuates to some extent conventional medication costs. However, the average costs of TCM medication (RMB1803/USD289), plus the use of conventional medications (RMB2087/USD335), meant that the total cost of medication for TCM user ischemic stroke inpatients was significantly higher (RMB4175/USD670) than TCM nonusers who only used conventional medication (RMB2716/ USD436). As a result, the extra medication cost of TCM users imposed a significant financial composition, roughly an additional $32.5 \%$, compared to the total medical cost of TCM nonusers. These data suggest that TCM was complementary, rather than a substitute, for conventional medical treatment for ischemic stroke inpatients.

Previous studies showed that TCM mainly played a role in the recovery period of ischemic stroke treatment. Although randomized clinical trials and animal studies have confirmed that TCM, specifically Chinese patent medicine, Chinese herbal medicine, and Chinese medicine injection, is 
effective in treating ischemic stroke [36-39], conventional recombinant tissue plasminogen activator is almost the only approved and available thrombolytic agent used at the onset of acute ischemic stroke $[40,41]$. As reported by both clinical trials $[25,40]$ and real-world studies $[42,43]$, the effectiveness and safety of TCM were mostly related to the ischemic stroke rehabilitation and recovery of motor function stages. There is no TCM medication to replace conventional thrombolytic medication during the onset of acute ischemic stroke.

TCM cost was positively associated with conventional medication cost after adjusting for the confounding factors. The use of TCM and conventional medicine altogether increased total inpatient cost among TCM users, revealing that TCM was complementary rather than an alternative for conventional medicine during the whole stroke treatment. This might be explained from both the demand and supply sides. From the demand side, patients in Asian countries, especially China, have a strong cultural belief in TCM, seeking additional TCM medication therapy and demanding TCM prescriptions [44, 45]. From the supply side, the drug markup policy and bonus system, which rewarded physicians based on the monetary values of drugs they prescribed, encouraged physicians to overprescribe drugs [46]. The zero-markup policy for conventional drugs, which was introduced into TCM hospitals from 2012, only reduced conventional drug prices marginally, including the cost of stroke-related drugs, by 2015 [6]. Since Chinese herbal medication was omitted from the zero-markup policy, partly to promote TCM medication use, we suspect that physicians might continue to overprescribe Chinese herbal medicine to pursue their own monetary rewards. We recommend that policymakers regulate the use and pricing of TCM as well as conventional medications to constrain any for-profit overprescribing. In addition, physician training and supervision should promote ethical use of TCM medications, advising patients on costs and benefits of TCM treatments.

A higher proportion of TCM users with ischemic stroke chose primary hospitals to receive medical services, consistent with a study from Taiwan [42]. For those who were admitted to primary hospitals, the total inpatient cost for TCM users was lower than TCM nonusers, though not statistically significant. Patients in the rehabilitation or recovery phase of ischemic stroke were more willing to seek medical treatment in primary hospitals [42].

Average length of hospital stay (ALOS) among TCM nonusers was significantly longer than TCM users in our study. On one hand, severe ischemic stroke patients, measured by longer hospital stays and a higher proportion of admission to tertiary hospitals (characterized by advanced clinical capability), may have had less opportunity to use TCM [43]. On the other hand, there was a higher proportion of UEBMI beneficiaries among TCM nonusers. UEBMI beneficiaries usually enjoyed higher reimbursement rate and more comprehensive insurance coverage, and thus they tended to seek more medical treatment and increase their length of stay [31]. Alternatively, the shorter TCM users'
ALOS may reflect the efficacy of TCM treatments. Future studies should investigate the role of ALOS in TCM user and TCM nonuser treatments.

There are a number of limitations to this study. First, the claims database could not directly provide evidence for the efficacy of TCM and clinical outcomes of treating ischemic stroke patients. Second, the severity of disease, an important factor influencing the use of TCM and its cost, was controlled using proxy indicators of the presence of comorbidities. Collecting data from patient clinical records would allow the severity of stroke to be assessed. Third, our study only analyzed the impact of prescription of TCM drugs on inpatient cost, including Chinese herbal medicine, Chinese patent medicine, and Chinese medicine injection. Other conventional TCM services like acupuncture and massage were attributed to nonpharmacy services in our study. Lastly, our study contained data in 2015 only. The post-2016 health care reforms, like the full implementation of the zerodrug markup policy, were likely to impact our results. While our sample is a representative sample of 2015 urban ischemic stroke patients, we are cautious about generalizing our results to other diseases.

\section{Conclusion}

Although TCM medication use lowered the cost of conventional medicine compared with TCM nonusers, the total inpatient cost for TCM users was significantly higher than TCM nonusers. The additional costs of ischemic stroke TCM users were due to the extra cost component of TCM medication imposed on top of conventional medication costs. We found that TCM medication costs were positively associated with conventional medication costs, suggesting that TCM mainly played a complementary, not substitute, role to conventional medicine in ischemic stroke treatment in mainland China.

\section{Abbreviations}

TCM: $\quad$ Traditional Chinese medicine

LOS: Length of stay

UEBMI: Urban Employee Basic Medical Insurance

URBMI: Urban Resident Basic Medical Insurance

IQR: Interquartile range.

\section{Data Availability}

The data that support the findings of this study are available from China Health Insurance Research Association but restrictions apply to the availability of these data, which were used under license for the current study and so are not publicly available. Data are however available from the authors upon reasonable request and with permission of the China Health Insurance Research Association.

\section{Ethical Approval}

Since the claims data the authors used were an anonymized and secondary database, human participants are not directly involved in the study. The Ethics Committee of Beijing 
University of Chinese Medicine (no. 2019BZHYLL0201) has waived the requirement of informed consent for the study. All methods were carried out in accordance with relevant guidelines and regulations.

\section{Consent}

The informed consent was exempted.

\section{Conflicts of Interest}

The authors stated that they have no conflicts of interest which might be perceived as posing a conflict or bias.

\section{Authors' Contributions}

Zhengwei Huang and Yan Jiang developed the research aims and drafted the manuscript; Zhengwei Huang and Weihan Zhao analyzed the data; Yong Yang, Stephen Nicholas, and Elizabeth Maitland revised the manuscript; Xuefeng Shi and Yan Jiang developed the research idea; Stephen Nicholas and Elizabeth Maitland helped interpret the data analysis; Yong Ma oversaw the data collection; and Xuefeng Shi collected the data and helped develop the idea and edited the manuscript. All authors reviewed the manuscript.

\section{Acknowledgments}

The authors acknowledge the China Health Insurance Research Association for providing access to their claims data.

\section{References}

[1] H. H. Kyu, D. Abate, K. H. Abate et al., "Global, regional, and national disability-adjusted life-years (DALYs) for 359 diseases and injuries and healthy life expectancy (HALE) for 195 countries and territories, 1990-2017: a systematic analysis for the global burden of disease study 2017," Lancet, vol. 392, no. 10159, pp. 1859-1922, 2018.

[2] G. A. Roth, D. Abate, K. H. Abate et al., "Global, regional, and national age-sex-specific mortality for 282 causes of death in 195 countries and territories, 1980-2017: a systematic analysis for the Global Burden of Disease Study 2017," Lancet, vol. 392, no. 10159, pp. 1736-1788, 2018.

[3] R. Luengo-Fernandez, M. Violato, P. Candio, and J. Leal, "Economic burden of stroke across Europe: a populationbased cost analysis," European Stroke Journal, vol. 5, no. 1, pp. 17-25, 2020.

[4] L. Liu, D. Wang, K. S. L. Wong, and Y. Wang, "Stroke and stroke care in China huge burden, significant workload, and a National priority," Stroke, vol. 42, no. 12, pp. 3651-3654, 2011.

[5] W. Wang, B. Jiang, H. Sun et al., "Prevalence, incidence, and mortality of stroke in China results from a nationwide population-based survey of 480687 adults," Circulation, vol. 135, no. 8, pp. 759-771, 2017.

[6] D. Zhu, X. Shi, S. Nicholas et al., "Medical service utilization and direct medical cost of stroke in urban China," International Journal of Health Policy and Management, vol. $\mathrm{x}$, no. $\mathrm{x}$, pp. 1-10, 2020.

[7] S.-W. Seto, D. Chang, A. Jenkins, A. Bensoussan, and H. Kiat, "Angiogenesis in ischemic stroke and angiogenic effects of
Chinese herbal medicine," Journal of Clinical Medicine, vol. 5, no. 6, p. 56, 2016.

[8] T. F. Hasan, N. Todnem, N. Gopal et al., "Endovascular thrombectomy for acute ischemic stroke," Current Cardiology Reports, vol. 21, no. 10, p. 112, 2019.

[9] L. Derex and T.-H. Cho, "Mechanical thrombectomy in acute ischemic stroke," Revue Neurologique, vol. 173, no. 3, pp. 106-113, 2017.

[10] S. H. Shah, R. Engelhardt, and B. Ovbiagele, "Patterns of complementary and alternative medicine use among United States stroke survivors," Journal of the Neurological Sciences, vol. 271, no. 1-2, pp. 180-185, 2008.

[11] M. F. Ali, A. F. Abdul Aziz, M. R. Rashid et al., "Usage of traditional and complementary medicine ( $\mathrm{T}$ and $\mathrm{CM}$ ): prevalence, practice and perception among post stroke patients attending conventional stroke rehabilitation in A teaching hospital in Malaysia," Medical Journal of Malaysia, vol. 70, no. 1, pp. 18-23, 2015.

[12] J. Durai Pandian, G. Toor, R. Arora et al., "Complementary and alternative medicine treatments among stroke patients in India," Topics in Stroke Rehabilitation, vol. 19, no. 5, pp. 384-394, 2012.

[13] S.-U. Park, S.-Y. Cho, J.-M. Park et al., "Integrative treatment modalities for stroke victims in Korea," Complementary Therapies in Clinical Practice, vol. 20, no. 1, pp. 37-41, 2014.

[14] Y.-I. Shin, C.-Y. Yang, M.-C. Joo, S.-G. Lee, J.-H. Kim, and M. S. Lee, "Patterns of using complementary and alternative medicine by stroke patients at two university hospitals in Korea," Evidence-Based Complementary and Alternative Medicine, vol. 5, no. 2, pp. 231-235, 2008.

[15] J. C. Chun, H. F. Pan, Y. C. Feng et al., "Utilization status and demand of community Chinese medical service in guangzhou," Chinese General Practice, vol. 13, no. 16, pp. 1742-1744, 2010.

[16] J. A. Lee, S.-W. Park, P. W. Hwang et al., "Acupuncture for shoulder pain after stroke: a systematic review," Journal of Alternative \& Complementary Medicine, vol. 18, no. 9, pp. 818-823, 2012.

[17] X.-Y. Yang, L.-Q. Wang, J.-G. Li, N. Liang, Y. Wang, and J.-P. Liu, "Chinese herbal medicine Dengzhan Shengmai capsule as adjunctive treatment for ischemic stroke: a systematic review and meta-analysis of randomized clinical trials," Complementary Therapies in Medicine, vol. 36, pp. 82-89, 2018.

[18] J. G. Zhao, C. H. Cao, C. Z. Liu et al., "Effect of acupuncture treatment on spastic states of stroke patients," Journal of the Neurological Sciences, vol. 276, no. 1-2, pp. 143-147, 2009.

[19] L. Arab, W. Liu, and D. Elashoff, "Green and black tea consumption and risk of stroke," Stroke, vol. 40, no. 5, pp. 1786-1792, 2009.

[20] Y.-B. Long and X.-P. Wu, "A meta-analysis of the efficacy of acupuncture in treating dysphagia in patients with a stroke," Acupuncture in Medicine, vol. 30, no. 4, pp. 291-297, 2012.

[21] N. Venketasubramanian, C. F. Lee, S. H. Young et al., "Prognostic factors and pattern of long-term recovery with MLC601 (NeuroAiD ${ }^{\mathrm{TM}}$ ) in the Chinese medicine NeuroAiD efficacy on stroke recovery-extension study," Cerebrovascular Diseases, vol. 43, no. 1-2, pp. 36-42, 2017.

[22] C.-C. Shih, C.-C. Liao, M.-F. Sun et al., "A retrospective cohort study comparing stroke recurrence rate in ischemic stroke patients with and without acupuncture treatment," Medicine (Baltimore), vol. 94, no. 39, Article ID e1572, 2015. 
[23] Y. C. Chen, J. S. Wu, S. T. Yang et al., "Stroke, angiogenesis and phytochemicals," Frontiers in Bioscience, vol. 4, pp. 599-610, 2012.

[24] Y. Chen, Y. Sun, W. Li et al., "Systems pharmacology dissection of the anti-stroke mechanism for the Chinese traditional medicine Xing-Nao-Jing," Journal of Pharmacological Sciences, vol. 136, no. 1, pp. 16-25, 2018.

[25] S.-Y. Han, Z.-Y. Hong, Y.-H. Xie, Y. Zhao, and X. Xu, "Therapeutic effect of Chinese herbal medicines for post stroke recovery: a traditional and network meta-analysis," Medicine (Baltimore), vol. 96, no. 49, Article ID e8830, 2017.

[26] S. Y. Su, C. H. Muo, and D. E. Morisky, "Use of Chinese medicine correlates negatively with the consumption of conventional medicine and medical cost in patients with uterine fibroids: a population-based retrospective cohort study in Taiwan," BMC Complementary and Alternative Medicine, vol. 15, p. 129, 2015.

[27] M.-Y. Tsai, W.-L. Hu, J.-H. Chiang et al., "Improved medical expenditure and survival with integration of traditional Chinese medicine treatment in patients with heart failure: a nationwide population-based cohort study," Oncotarget, vol. 8, no. 52, pp. 90465-90476, 2017.

[28] L. Zhang, H. Wang, Q. Li, M.-H. Zhao, and Q.-M. Zhan, "Big data and medical research in China," BMJ, vol. 360, Article ID j5910, 2018.

[29] Q. Meng, H. Fang, X. Liu, B. Yuan, and J. Xu, "Consolidating the social health insurance schemes in China: towards an equitable and efficient health system," The Lancet, vol. 386, no. 10002, pp. 1484-1492, 2015.

[30] H. Fang, K. Eggleston, K. Hanson, and M. Wu, "Enhancing financial protection under China's social health insurance to achieve universal health coverage," BMJ, vol. 365, Article ID 12378, 2019.

[31] Y. Yang, X. Man, S. Nicholas et al., "Utilisation of health services among urban patients who had an ischaemic stroke with different health insurance-a cross-sectional study in China," BMJ Open, vol. 10, no. 10, Article ID e040437, 2020.

[32] WHO, International Statistical Classification of Diseases and Related Health Problems 10th Revision, ICD-10, WHO, Geneva, Switzerland, 2016, http://www.who.int/classifications/ icd/ICD10Volume2_en_2010.pdf.

[33] R. Ding, D. Zhu, Y. Ma, X. Shi, and P. He, "Comparison of health service use and costs in stroke with and without comorbidities: a cross-sectional analysis using China urban medical claims data," BMJ Open, vol. 10, no. 12, Article ID e037032, 2020.

[34] R. L. Sarnat and J. Winterstein, "Clinical and cost outcomes of an integrative medicine IPA," Journal of Manipulative and Physiological Therapeutics, vol. 27, no. 5, pp. 336-347, 2004.

[35] C.-C. Tu, C.-S. Li, C.-M. Liu, and C.-C. Liu, "Comparative use of biomedicine and Chinese medicine in Taiwan: using the NHI Research Database," Journal of Alternative and Complementary Medicine, vol. 17, no. 4, pp. 339-346, 2011.

[36] J.-J. Lee, W.-H. Hsu, T.-L. Yen et al., "Traditional Chinese medicine, Xue-Fu-Zhu-Yu decoction, potentiates tissue plasminogen activator against thromboembolic stroke in rats," Journal of Ethnopharmacology, vol. 134, no. 3, pp. 824-830, 2011

[37] S. S. Wu, J. Hao, W. Y. Gao, B. Zhou, S. L. Man, and L. Q. Huang, "Neuroprotective effect and chemical composition of traditional Chinese medicines, Zha-Chong-Shi-SanWei Pill, against ischemic stroke on middle cerebral ischemia occlusion in rats," Latin American Journal of Pharmacy, vol. 32, no. 7, pp. 1020-1026, 2013.
[38] M. Yu, Z.-J. Sun, L.-T. Li, H.-Y. Ge, C.-Q. Song, and A.-J. Wang, "The beneficial effects of the herbal medicine Dihuang-yin-zi (DHYZ) on patients with ischemic stroke: a Randomized, Placebo controlled clinical study," Complementary Therapies in Medicine, vol. 23, no. 4, pp. 591-597, 2015.

[39] H. Zhao, D. Yang, M. Shi, J. Liang, X. Yang, and S. Jin, "Clinical efficacy and safety of Traditional Chinese Medicine combined with Western Medicine in patients with diabetic acute ischemic stroke," Journal of Traditional Chinese Medicine, vol. 34, no. 2, pp. 145-149, 2014.

[40] P. Sandercock, J. M. Wardlaw, R. I. Lindley et al., "The benefits and harms of intravenous thrombolysis with recombinant tissue plasminogen activator within $6 \mathrm{~h}$ of acute ischaemic stroke (the third international stroke trial [IST-3]): a randomised controlled trial," Lancet, vol. 379, no. 9834, pp. 2352-2363, 2012.

[41] National Institute of Neurological Disorders and Stroke rt-PA Stroke Study Group, "Tissue plasminogen activator for acute ischemic stroke," New England Journal of Medicine, vol. 333, no. 24, pp. 1581-1587, 1995.

[42] W.-S. Chen, H.-C. Hsu, Y.-W. Chuang et al., "Predictors for the use of traditional Chinese medicine among inpatients with first-time stroke: a population-based study," Bmc Complementary Medicine and Therapies, vol. 20, no. 1, p. 244, 2020.

[43] C. C. Liao, J. G. Lin, C. C. Tsai et al., "An investigation of the use of traditional Chinese medicine in stroke patients in taiwan," Evidence-based Complementary and Alternative Medicine: eCAM, vol. 2012, Article ID 387164, 2012.

[44] L. Jin, "From mainstream to marginal? Trends in the use of Chinese medicine in China from 1991 to 2004," Social Science and Medicine, vol. 71, no. 6, pp. 1063-1067, 2010.

[45] Y. Ma, Y. Liu, H.-M. Fu et al., "Evaluation of admission characteristics, hospital length of stay and costs for cerebral infarction in a medium-sized city in China," European Journal of Neurology, vol. 17, no. 10, pp. 1270-1276, 2010.

[46] D. Zhu, X. Shi, S. Nicholas, Q. Bai, and P. He, "Impact of China's healthcare price reforms on traditional Chinese medicine public hospitals in Beijing: an interrupted timeseries study," BMJ Open, vol. 9, no. 8, Article ID e029646, 2019. 